\title{
Micro- and Nano-Antennas for Light Detection
}

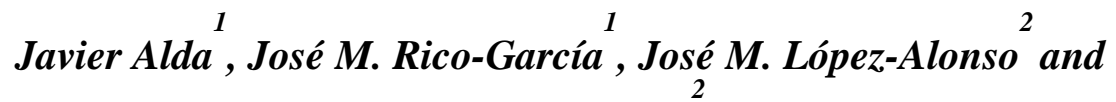 \\ Glenn Boreman
}
${ }^{(1)}$ Applied Optics Complutense Group. University Complutense of Madrid.

School of Optics. Av. Arcos de Jalón s/n. 28037 Madrid. Spain.

(2) College of Optics \& Photonics / CREOL. University of Central

Florida.

Central Florida Blvd. 4000. Orlando. Florida (USA).

\begin{abstract}
Antenna-coupled optical detectors, also named as optical antennas, are being developed as detection devices with micro- and nano-scale features for their use in the millimetre, infrared, and visible spectral range. They are optical components that couple the electromagnetic radiation in the visible and infrared wavelengths in the same way that radioelectric antennas do at the corresponding wavelengths. Optical antennas show polarization dependence, tuneability, and rapid time of response. They also cann be cosndiered as point detectors and directionally sensitive elements. So far, these detectors have been operated in the mid-infrared with positive results in the visible. The measurement and characterization of optical antennas requires the use of an experimental set-up with nanometric resolution. On the other hand, a computational simulation of the interaction between the material structures and the incoming electromagnetic radiation is needed to explore alternative designs of practical devices. In this contribution we will present the concept of optical and infrared antennas, and some experimental results of their performance, along with the experimental set-up arranged for their characterization in the visible.
\end{abstract}

E-mail: j.alda@fis.ucm.es 


\section{Introduction}

Antennas have played an essential role since the very beginning of Electromagnetism. Even one of the simplest and first designs, the dipole antenna, is being used nowadays because of its easy implementation and characteristics. In telecom applications the need for larger and larger bandwidths has demanded the use of higher and higher frequencies of the supporting electromagnetic waves. The use of high-frequency radio waves and the inclusion of microwave radiations improved the performance and capacity of the previous links. At the same time, the antenna design developed more sophisticated layouts that have been successfully applied. From our point of view, an important leap was done with the design of planar antennas structures. They can be fabricated with imprinting and thin-film technologies on an appropriate substrate. In the radioelectric spectrum, the shrinking in wavelength has been associated with the downscaling of antenna structures. Fortunately, the available fabrication techniques and the good radiation-metal interaction have allowed the realization and demonstration of the devices at shorter and shorter wavelengths. However, before antennas could reach optical frequencies in a reliable and practical way, the use of semiconductor detectors made possible the development of reliable lightwave links in free space and along dielectric waveguides (optical fibres). These optical networks use the available detectors in the optical range mainly based on semiconductor technology. The efforts to improve the performance of these detectors, and the positive results obtained from these efforts, have deprived the need to look back toward the antenna designs and make them work also in the optical band.

At the same time, but working in a very distant field of application, researchers dealing with time standards and astronomy were developing new solutions based in antenna-like detectors. One of the results of these efforts is a new class of optical detectors that we named as optical antennas. They are derived from the whisker diodes [1,2] first used for frequency-conversion and frequency-multiplication chains [3]. The uniqueness of this application has hidden the potential of the design for some other broader uses that were anticipated by several research groups $[4,5,6]$. A step forward was made when the concept of an antenna for the infrared radiation was totally included in the analysis and design of such devices, specially when taking into account the specific properties of the interaction of light and metals at optical frequencies. Another important progress in the realization of practical devices using antennacoupled detectors was their fabrication by using electron-beam lithography for the definition of micron and submicron structures [7,8] (see Figure 1). The smoothness of the finished metal structures, along with the spatial resolution of this manufacture technique, has been essential to the feasibility of the devices and to their performance even at visible frequencies [9]. 
In telecom applications, the detection devices typically used for light detection are well based on the semiconductor technology. Successive generations of semiconductor detectors have improved their responsivity, allowing long-haul lines, and their speed of response with the corresponding increase in bandwidth. For electromagnetic waves at lower frequencies (in the microwave and radioelectric portions of the spectrum), the detection mechanism is quite different. Antennas are used here to transform the energy contained in waves propagating in free-space into guided electromagnetic signals fed to a dedicated electronics. This electronics is in charge of the conversion of received signals into a more usable form able to be transmitted along cables and transmission lines. "Optical antennas" are in between the two previous approaches of detection mechanisms: semiconductor photodetectors and radioelectric antennas. They are intended to work in the optical range and they include metallic structures that couple the radiation towards a transductive element. With this strategy, "optical antennas" benefit from the best of both worlds: the bandwidth of the optical communications links and the selective properties in polarization, frequency, and direction related with the antenna designs.

Another field of application for optical antennas originates from the field of nano-optics and nano-photonics. We have here a branch of nanometerscale science and technology ("nanoscience") dealing with the optical properties of material structures less than $100 \mathrm{~nm}$ in size (and down to molecular dimensions) as well as optical processes occurring on that scale [10,11,12]. Among those, the excitation and properties of surface plasmon polaritons play a prominent role [13]. Surface plasmon polaritons are collective oscillations in the density of the electron gas of metals. At optical frequencies, these oscillations are of primary importance for the induction of antenna currents as well as for the propagation of signals along wires. The existence of polaritons is closely related to the poor conductivity of most metals at optical frequencies but it also opens up opportunities for the design of electric components that will be specific for the optical regime. Therefore, the combination of antenna resonances and plasmons resonances hold promise for giant localized field enhancement. Optical frequency radiation can be confined to dimensions considerably less than $100 \mathrm{~nm}$ only by material structures of similar size, e.g. small apertures and scattering particles. Such structures have been used already for optical imaging beyond the diffraction limit by means of scanning near-field optical microscopy [14]. In a generalized sense, such structures are antennas, the confinement volume corresponding to the feed point region of the antenna. Apertures and small spheres, however, are not necessarily optimal shapes for the field confinement needed in optical communications networks. For this reason, scientists studying nanoscience topics recently have become interested in the properties of radiowave antennas and their potential for downscaling into the optical regime [15]. 


\section{The Concept of an Optical Antenna:}

An optical antenna is the combination of two elements. The metallic structure in charge of the coupling of the electromagnetic field, and the rectifying element that converts the energy trapped in currents along the antenna into a manageable signal [16]. Therefore, an optical antenna is a light detector. The electromagnetic builds up a pattern of oscillating currents within the metal structure. At optical frequency, the metal structures show important losses. However, the currents surviving in the metal are strong enough to produce signals readable by a dedicated electronics. From the antenna design principles, the size of the metallic structures are scaled with the wavelength, and typical features are a fraction of the detected wavelength. This makes optical antennas a point-like detector. The transducer element is responsible for the rectification of the currents flowing across the metal structures. As far as there is not possible a conversion into an electric signal carrying information of the frequency of the optical signal, the detection only retrieves the value of the irradiance falling onto the detection area. The physical mechanisms involved in this rectification process are of several kinds. Among them, we have experienced the use of microbolometers and metal-oxide-metal (MOM) junctions. The former transducers are easier to fabricate than MOMs, having a slower response. However, microbolometers are fast enough to be used in image-forming systems.

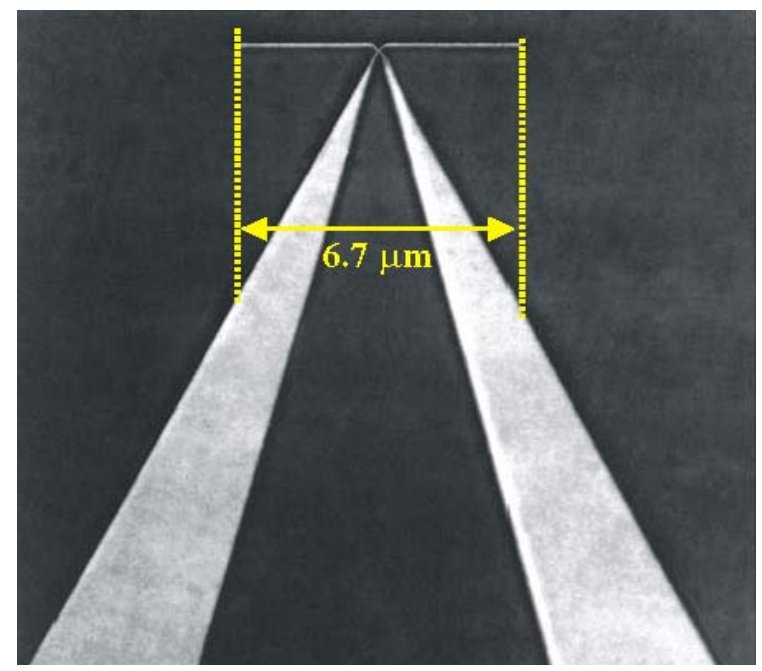

Fig. (1): Photography taken with an electron microscope showing the arrangement of an optical antenna designed to resonate in the infrared, and having a dipoleantenna design. The dipole is formed by the two metallic arms located at the top of the picture. The V-shaped metallic strips are connection lines in charge of the retrieval of the signal detected by the antenna and rectified by a MetalOxide-Metal diode located at the intersection point. 
Taking the previous design into account we may say that optical antennas stands for a new class of light detector, or as a metallic structure that couples electromagnetic radiation at optical frequencies. On the other hand, we also can state what is not an optical antenna. It is not a simple downscaled version of a radioelectric antenna because of the very different behaviour of metal at optical frequencies with respect to the behaviour of their counterparts at radio-band and microwave-band. Due to the maturity of semiconductor detectors, optical antennas are not a menace for them. Optical antennas will find its application niche in those areas where their outstanding characteristics in polarization sensitivity, and point-like footprint are preferred and appreciated. The high frequency of the optical radiation and the need to extract electric signals manageable by post-processing electronics makes not possible a phase detection of the incoming radiation. Finally, so far there have not been positive results reporting the emission of optical radiation by optical antennas structures. In figure 1 we show an electron micrography of a dipole optical antenna designed for the infrared. The dipole is materialized by the horizontal structure having a total length of $6.7 \mu \mathrm{m}$. The transductor is located in the junction appearing in the center of the dipole. This case corresponds with a MOM diode. The V-shaped metallic structures are the connection lines used to extract the rectified signal from the antenna. The metallic structure has been written on a $\mathrm{Si} / \mathrm{SiO}_{2}$ wafer. The light is inciding perpendicularly to the plane represented in the picture. It can be coming from the air or from the substrate [17].

Different designs of optical antennas have been proposed mimicking some of the proved layouts already working in the microwave and radioelectric design. In Figure 2 we show several possible designs fabricated with electronbeam lithography.
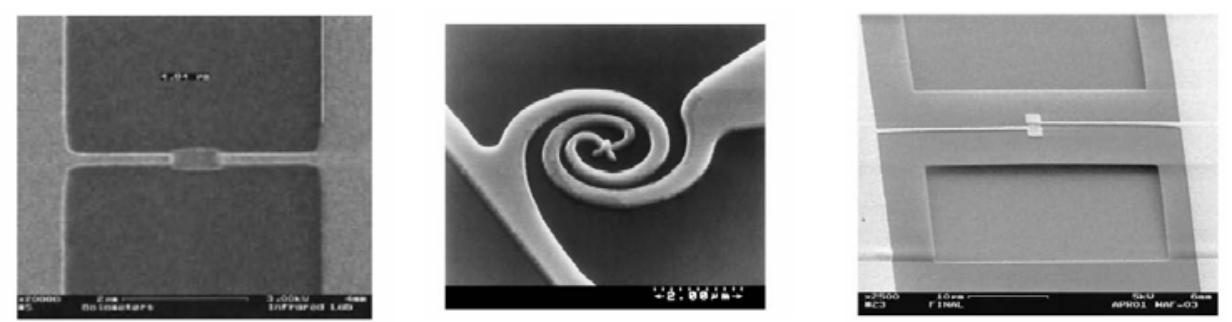

Fig. (2): Different types of optical antennas written by electron beam lithography on $\mathrm{Si}$ wafers. We can see a dipole antenna (left), an asymmetric spiral antenna (center), and a patch antenna on a moving bridge (right). The physical mechanism for the transducer is a microbolometer for the left and righ antennas, and a MOM diode for the central one. 


\subsection{Properties of an optical antenna:}

In this subsection we can itemize the special properties of an optical antenna.

Subwavelength size of the responsivity map. The area containing about the $90 \%$ of the spatial responsivity of an optical antenna has been measured to be about the square of the detected wavelength, both in the infrared and visible spectrum [18]. Figure 3 shows the response of a dipole antenna at infrared and visible frequencies $[9,18]$
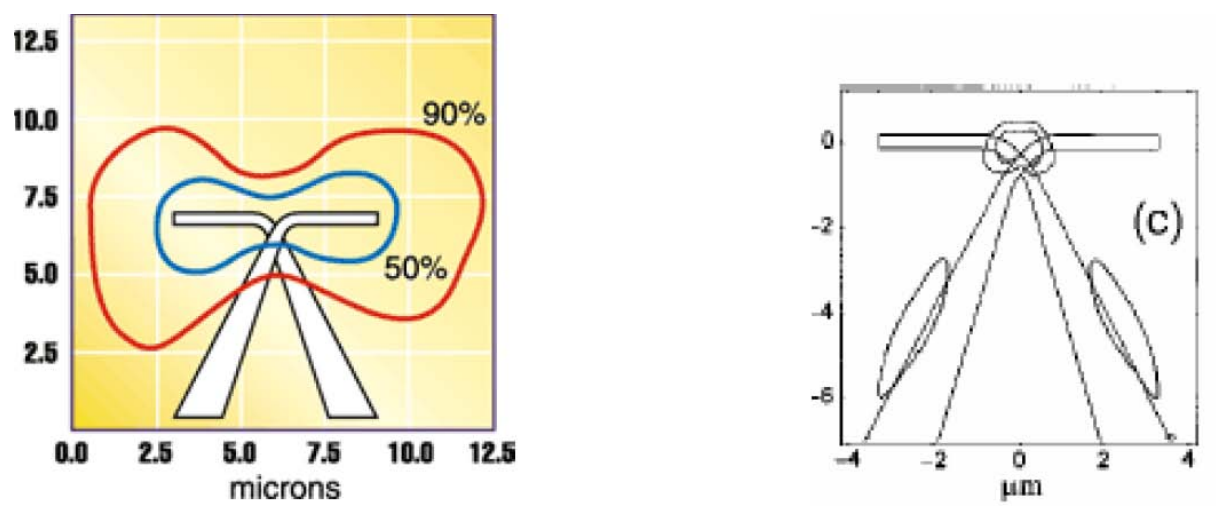

Fig. (3): spatial response of a dipole optical antenna in the infrared (left) and the same antenna in the visible (right)

Response time. The tiny volumes of the rectifying element, about 0.02 $\mu \mathrm{m}$ allows to obtain a very fast response. This is specially important when using microbolometers because the thermal inertial is directly related with the volume of bolometric material. Preliminary estimations of the response time for non-optimised devices are about 100 ns [19]. On the other hand, when MOM diodes are used as rectifying elements, the response of the antenna is typically limited by the transmission lines and the associated electronics. The intrinsic response time of the physical mechanism underlying the detection is about about $10^{-14,-15}$ s [20].

Polarization sensitivity. The antennas are intrinsically polarization sensitive elements, in the same way as their radioelectric versions $[9,18,21]$ (see figure 4). 

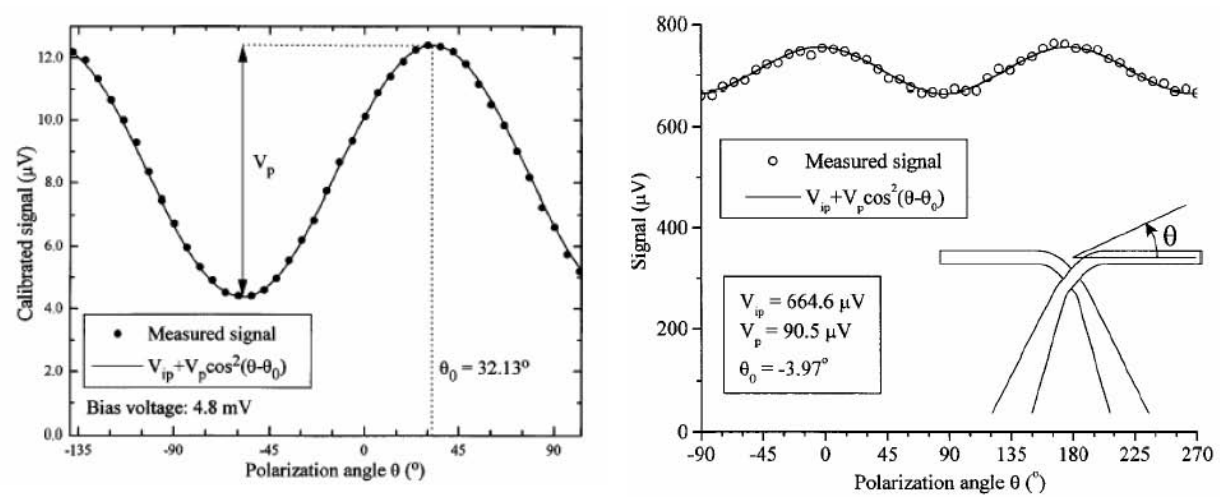

Fig. (4): Polarization sensitivity of a dipole optical antenna in the infrared (left) and in the visible (right).

Tunning. The behaviour of metals at optical frequency limits the tuneability of optical antennas because of the losses induced in the metallic structure. This capability may compromise fine tuning of the antennas. However, some clever electromechanical layouts may overcome this problem and produce a reasonable tunning of the devices.

Directionality. Antennas are direction selective elements per se. This property is strongly dependent on the geometry of the antenna [22]. In figure 5 we show the results obtained for several dipole antennas by changing the dipole length.

(a)

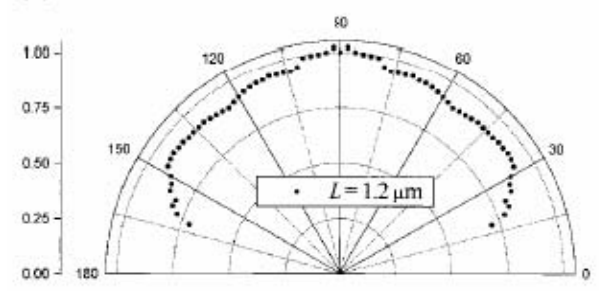

(c)

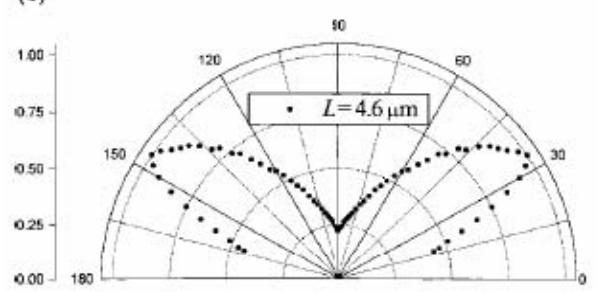

(b)

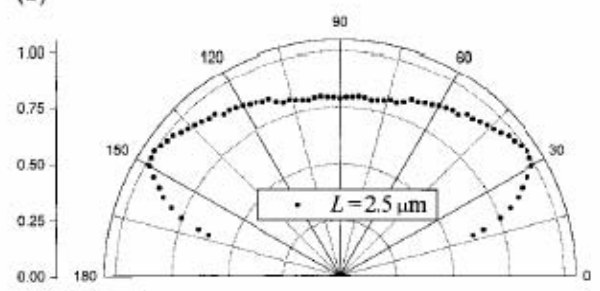

(d)

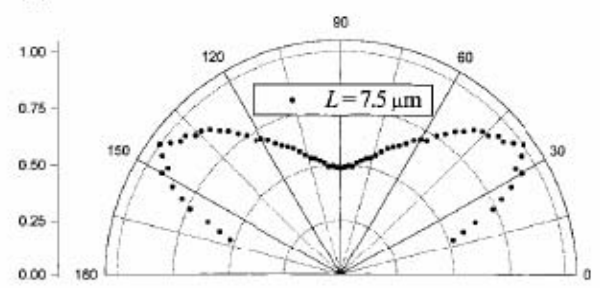

Fig. (5): Directional sensitivity of an dipole optical antenna as a function of the dipole length for the infrared at $10.6 \mu \mathrm{m}$. 
Integrability. As far as the fabrication tools and substrates are the same used for microelectronics, some specific designs of "Optical antennas" can be monolithically integrated with associated electronics, micro-mechanical systems, and integrated optics [23,24]. In figure 6 we show the use of Fresnel zone plates specifically designed to improve the responsivity of the optical antenna.
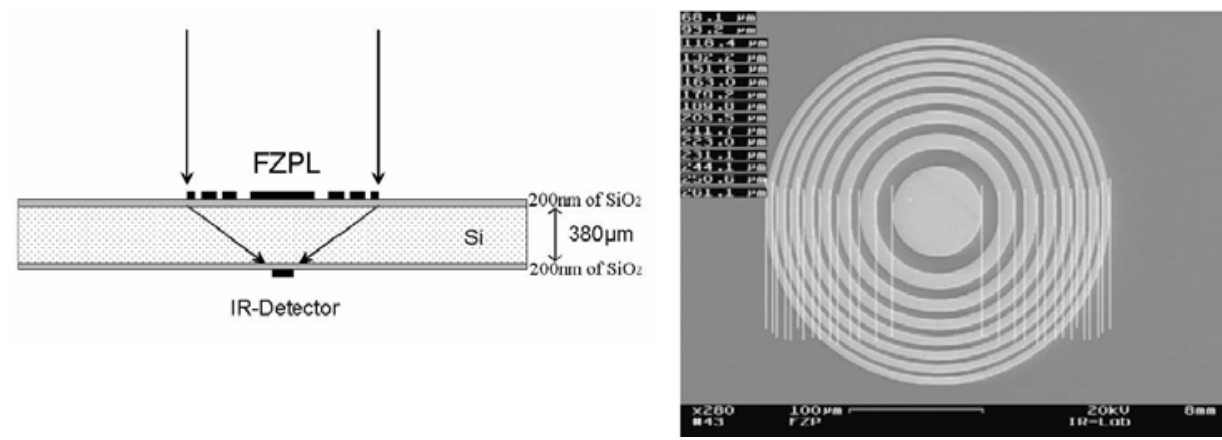

Fig. (6): Optical antennas may include diffractive optical elements to enhance their responsivity and detectivity. The Fresnel zone plate fabricated here made possible a gain in the responsivity of $\mathrm{x} 160$.

Room temperature operation. Optical antennas working in the infrared, even in the far infrared band, do no need auxiliary cooling subsystems. This advantage is very much appreciated when comparing optical antennas with the conventional detection technologies that use sophisticated cryogenic equipment to reduce the noise of the detectors.

Some of these properties are intrinsically related with the antenna design and can not be performed by semiconductor detectors. The most promising application of optical antennas will make full use of the advantage offered by the most appealing intrinsic properties $[25,26]$.

\section{Measurement and Characterization:}

The characterization of this novel type of device is of interest for evaluating comparatively the performance of optical antennas with respect to any other types of light detectors. The typical figures of merit in optical detection are evaluated using their well established definitions. These parameters are: responsivity, time of response, normalized detectivity, and noise 
equivalent power. On the other hand, the special properties of optical antennas make necessary to characterize some other parameters. They are the spatial map of the responsivity, the polarization sensitivity and the frequency response. In any case, the measurement of optical antennas is a compelling task dealing with sub-wavelength measurements [27]. Besides, the response of the device is, sometimes, having a non-negligible contribution of different kind of noises. These sources of noise have to be identified and, if possible, filtered out. One of them is the presence of $1 / \mathrm{f}$ noise. This noise is easily reduced by modulating the optical input and by detecting synchronously the response of the optical antenna.

\subsection{Responsivity Mapping:}

Among all these previous parameters, probably the most difficult to measure is the spatial responsivity map [18]. What we mean with this characterization is to know how is the map of the response of the device, $R(x, y)$, for a given irradiance distribution, $I(x, y)$, falling at different locations on the antenna. When scanning the optical antenna under a given light beam, the signal obtained by the device can be seen as the convolution of the irradiance distribution and the responsivity map of the antenna. This can be written as,

$$
\mathrm{S}(x, y)=\mathrm{R}(x, y) * \mathrm{I}(x, y)
$$

The characterization of the responsivity map requires the accurate measuring of the signal when the antenna scans a given light beam distribution, and a post-processing to implement the deconvolution (see Figure 7).

One of the most important pieces of the method is the knowledge of the light beam distribution. This distribution corresponds with a very focused and low aberrated beam. The tiny size of the beam makes no possible its characterization by imaging this irradiance on a detection array. Therefore, some integrated methods are necessary, adding more complexity to the problem. The whole experimental chain of the characterization of the spatial response has been recently analyzed using multivariate statistical techniques based on the principal component analysis [28,29]. 

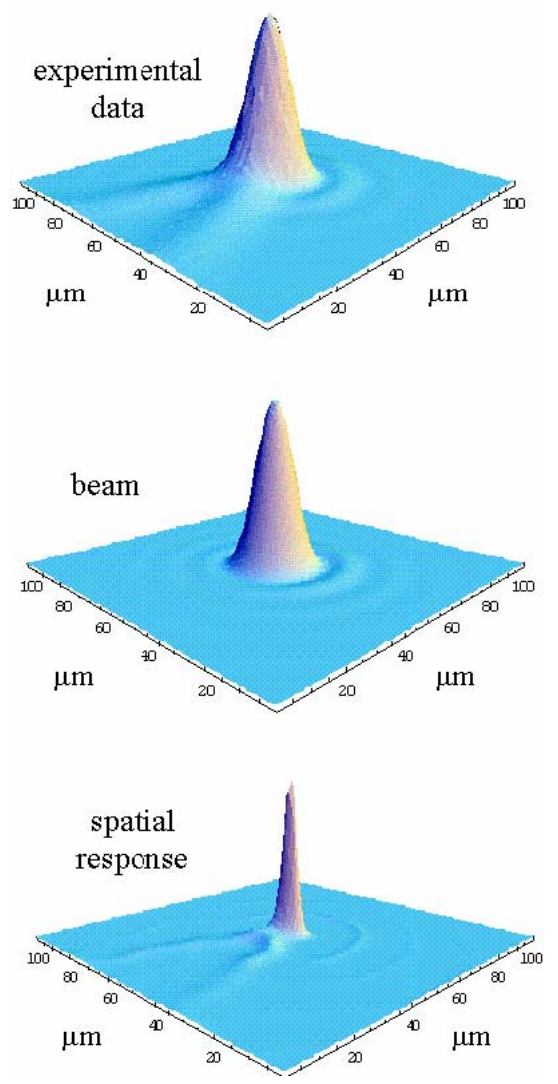

Fig. (7): Graphical representation of the elements involved in the characterization of the responsivity map of a given optical antenna. The experimental data are obtained after scanning the detector under a given beam irradiance distribution as the one represented in the middle. Finally, a convolution algorithm is used to retrieve the spatial response of the antenna.

\section{Conclusions:}

In this paper we have made a brief presentation of a new kind of optical detector that we have named as optical antennas. They use the same type of fundamental mechanism than the one already proved by the radioelectric antennas. The metallic structures of optical antennas couple the electromagnetic radiation and excite electric currents that are rectified and converted into a manageable signal by a transductor element located at the feed point of the antenna. Optical antennas can be shape in a great variety of forms and configurations, sometimes mimicking their radiolectric counterparts, and some other times generating new designs and elements able to enhance the appealing properties of the optical antennas. 
The most interesting characteristics of optical antennas are: their pointlike character (actually, they could be considered as the optical detectors having the minimum size); their polarization sensitivity; the broad-band response in wavelength; the fast time of response when using metal-oxide-metal; their integrability with read-out electronics, and diffractive optics; and their natural operation at room temperature. Among those characteristics that need to be optimized we may find their low responsivity, the attenuation due to thermal effects, the aging of the devices, and their sensibility to electric static discharges [30]. Most of these problems can be addressed by an appropriate change in the design and fabrication protocols.

Summarizing the previous remarks we may conclude that optical antennas represent a portion of light detection area where technologic and fundamental problems need to be taken into account simultaneously. The research on this topic will benefit from advances in the precise knowledge of the interaction between electromagnetic radiation and matter, and also on the expertise in the design, fabrication and testing of the manufactured devices. The fundamental problems usually need the assistance of computational electromagnetism, and the practical issues always make use of the improvement in the accuracy of measurement techniques and the sharp contributions of scientists and engineers working on this state-of-the-art technology. Some of the research results obtained in this field can be obtained from the following webpages: http://www.ucm.es/info/euoptica/org/pagper/jalda/jalda.htm, and http://ir.creol.ucf.edu (accessed May 2005).

\section{Acknowledgments:}

Most of the work presented in this paper has been possible due to the economic support of several research projects. Among them, we would like to acknowledge the participation of the Ministerio de Ciencia y Tecnología of Spain through the project TIC2001-1259, and to the Consejería de Educación of the Comunidad de Madrid (Spain) through the project GR/MAT/0497/2004. The collaboration between the University Complutense of Madrid, and the University of Central Florida has been possible thanks to a collaboration agreement among these two institutions. 


\section{References:}

1. Bor-Iong Twu, S. E. Schwarz, Applied Physics Letters 26(12), 672 (1975).

2. O. Acef, L. Hilico, M. Bahoura, F. Nez, P. De Natale, Optics Communications 109, 428-434, (1994).

3. K. M. Evenson "Frequency measurements from the microwave to the visible, the speed of light, and the redefinition of the meter", Quantum Metrology and Fundamental Physical Constants, Ed.: P.H. Cutler \& A.A. Lucas, Nato ASI Series B: Physics Vol. 98, 181 (1983).

4. D. B. Rutledge, M. S. Muha, IEEE Transaction on Antennas and Propagation, AP-30, 535 (1982).

5. C. R. Brewitt-Taylor, D. J. Gunton, H. D. Rees, Electronic Letters, 17, 729 (1982).

6. E. N. Grossman, J. E. Sauvageau, D. G. McDonald, Applied Physics Letters, 59, 3225 (1991).

7. I. Wilke, Y. Oppliger, W. Herrmann, F.K. Kneubühl, Applied Physics A 58, 329 (1994).

8. C. Fumeaux, W. Herrmann, F.K. Kneubühl, H. Rothuizen, Infrared Physics \& Technology 39, 123 (1998).

9. C. Fumeaux, J. Alda, G.D. Boreman, Optics Letters 24, 1629 (1999).

10. B. Hecht, B. Sick, U.P. Wild, V. Deckert, R. Zenobi, O. J. F. Martin and D.W. Pohl, J. Chem. Phys. 112, 7761 (2000).

11. Michael A. Paesler, Patrick J. Moyer, "Near-field optics: theory, instrumentation, and applications", Wiley-Interscience, New York (1996).

12. S. Kawata, "Nano-Optics", Springer series in optical sciences 84, Springer, Berlin, (2002).

13. S. Kawata (Ed.), "Near Field Optics and Surface Plasmon Polaritons", Springer Series in Applied Physics 81, Springer Verlag, Berlin/Heidelberg (2001).

14. R.C. Dunn, "Near-field scanning optical microscopy" Chem. Rev. 99 (10), 2891 Oct, 1999.

15. D. W. Pohl, "Near field optics seen as an antenna problem", in "Near-Field Optics: Principles, Applications" / The Second Asia-Pacific Workshop on Near Field Optics, Beijing, China October 20 -23, 1999", EDITOR M.Ohtsu, X. Zhu 9 (2000).

16. G. Boreman, Optical Engineering Mag. 2, 47 (2002)

17. J. Alda, C. Fumeaux, M. Gritz, D. Spencer, G. Boreman, Infrared Physics and Technology, 41, 1 (2000).

18. J. Alda, C. Fumeaux, I. Codreanu, J.A. Schaefer, G. D. Boreman, Applied Optics 38, 3993 (1999).

19. F. J. Gonzalez, M. A. Gritz, C. Fumeaux, G. D. Boreman, International Journal of Infrared and Millimeter Waves 23, 785 (2002).

20. E. H. Hauge, J. A. Støvneng, Reviews of Modern Physics 61, 917 (1989). 
21. G. D. Boreman, C. Fumeaux, W. Herrmann, F. K. Kneubühl, H. Rothuizen, Optics Letters 23, 1912 (1998).

22. C. Fumeaux, M. A. Gritz, I. Codreanu, W. L. Schaich, F. J. Gonzalez, G. D. Boreman, Infrared Physics and Technology 41, 271 (2000).

23. F. J. González, J. Alda, B. Ilic, G. D. Boreman, Applied Optics, 43, 6067 (2004).

24. J. M. Rico-García, J. M. López-Alonso, B. Lail, G. Boreman, J. Alda, Proceedings SPIE, 5612, 216 (2004)

25. F. J. González, B. Ilic, J. Alda, G. Boreman, IEEE Journal of Selected Topics in Quantum Electronics, 11, 117 (2005)

26. J. Alda, J. M. Rico-García, J. M. López-Alonso, G. Boreman, Nanotechnology, 16, S230 (2005).

27. J. Alda, J. M. López-Alonso, J. M. Rico-García, J. Zoido, G. Boreman, Proceedings SPIE, 5407, 226 (2004)

28. J. M. López-Alonso, B. Monacelli, J. Alda, G. D. Boreman. Optical Engineering (in press) (2005)

29. J. M. López-Alonso, B. Monacelli, J. Alda, G. D. Boreman, Applied Optics (in press) (2005)

30. J. González, C. Fumeaux, J. Alda, G. Boreman, Microwave and Optical Technology Letters, 26, 291 (2000). 